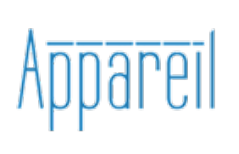

Appareil

2| 2008

Autour de Simondon

\title{
Évolution technique et objectivité technique chez Leroi-Gourhan et Simondon
}

Xavier Guchet

\section{(2) OpenEdition}

1 Journals

\section{Édition électronique}

URL : http://journals.openedition.org/appareil/580

DOI : 10.4000/appareil.580

ISSN : 2101-0714

Éditeur

MSH Paris Nord

Référence électronique

Xavier Guchet, «Évolution technique et objectivité technique chez Leroi-Gourhan et Simondon »,

Appareil [En ligne], 2 | 2008, mis en ligne le 11 septembre 2008, consulté le 30 juillet 2020. URL : http:// journals.openedition.org/appareil/580 ; DOI : https://doi.org/10.4000/appareil.580

Ce document a été généré automatiquement le 30 juillet 2020.

\section{(c) (i) (3)}

Appareil est mis à disposition selon les termes de la Licence Creative Commons Attribution - Pas d'Utilisation Commerciale - Pas de Modification 4.0 International. 


\title{
Évolution technique et objectivité technique chez Leroi-Gourhan et Simondon
}

\author{
Xavier Guchet
}

\section{Introduction. L'évolution technique, simple métaphore?}

1 La première difficulté que l'on rencontre dans l'effort pour élucider la signification de la notion d'évolution technique, c'est qu'il faut se convaincre que cette notion est intéressante. Une notion est un concept intéressant si elle rend possible une mise en ordre de l'expérience et, par-là, une production de connaissances nouvelles. Or, il n'est pas du tout sûr que la notion d'évolution technique soit de nature à permettre cette production de connaissances. Il semble même qu'en opérant un rapprochement purement métaphorique entre le changement technique et l'évolution des êtres vivants, cette notion empêche la production d'une connaissance véritable des techniques et de leur devenir. C'est en tout cas le principal grief qui est adressé à l'idée d'évolution technique par ses détracteurs. Leur critique est en substance que ce rapprochement purement métaphorique, sans valeur épistémologique, avec le domaine biologique a pour conséquence de naturaliser les objets techniques et de les couper de l'ordre humain des significations. Les objets techniques, ainsi naturalisés et coupés du monde humain, deviennent par conséquent inconnaissables en tant que productions sociales, historiques, culturelles et symboliques.

On ne peut pas leur donner complètement tort. Que peut-on attendre en effet, en termes de possibilités de connaissances, d'un propos comme celui de M. Minski, l'un des pionniers de l'Intelligence Artificielle aux États-Unis dans les années soixante : « au commencement les machines n'avaient que de simples griffes. Mais bientôt elles seront dotées de fantastiques articulations ${ }^{1}$ » (Minsky prophétisait de spectaculaires réalisations dans le domaine de l'IA) ? La machine devient une sorte d'être vivant qui 
évolue vers davantage de complexité, des performances plus importantes etc. Thème inusable en vérité, que l'on retrouve par exemple aujourd'hui chez les promoteurs les plus convaincus des nanotechnologies:

au cours des âges, les mutations et la sélection des gènes ont recouvert le monde de prairies et d'arbres, d'insectes, de poissons et d'hommes. Plus récemment, d'autres objets sont apparus et se sont multipliés : les outils, les maisons, les avions et les ordinateurs. Et comme les molécules d'ARN sans vie, ce matériel a évolué.

3 Deux exemples semblent donner raison à ceux qui refusent toute valeur de connaissance à ce concept. Le premier exemple est une affiche de la RATP que B. Latour n'a pas manqué de railler'2. L'affiche présente une succession d'autobus, du plus ancien au plus récent, en donnant à penser que les bus se transforment comme le font les espèces biologiques, c'est-à-dire par une adaptation toujours plus fine à leur environnement. « Darwin avait raison » précise l'affiche. L'établissement d'une filiation entre autobus ne s'appuie cependant sur aucun élément de connaissance scientifique et technique. C'est pourquoi Latour tourne en dérision ce qu'il appelle cette «biologie de pacotille » et fait dire au professeur Norbert $\mathrm{H}$. qu'un autobus, «à moins que je ne sois pas doué pour ces choses-là, n'a pas d'organes sexuels. Le R-312, malgré l'affiche, ne descend pas du Chausson APU 53 comme l'homme descend du singe ${ }^{3} »$. Nous le savons, l'homme ne descend pas du singe: le concept d'évolution technique repose donc sur une biologie anti-scientifique au possible, une "biologie de pacotille» en effet qui en est restée au mythe de l'homme singe et, pourquoi pas, à la recherche du chaînon manquant.

Le second exemple est tiré d'un ouvrage de T.Gaudin ${ }^{4}$. Il s'agit d'une planche censée mettre en évidence l'évolution des wagons de train. Cette illustration est très semblable à celle des autobus, elle ne s'appuie sur aucun élément de connaissance scientifique et technique, ce qui n'empêche pas T. Gaudin d'affirmer le caractère scientifiquement justifié du constat d'évolution fait par Lorenz au xix siècle :

l'évolution des objets techniques ressemble à celle des êtres vivants... On reconnaît dans la technique la même morphogenèse que celle qu'observent les paléontologues : des organes leur poussent, d'autres s'atrophient. [L'exemple des wagons de train] montre bien l'absence de planification préalable dans l'évolution de ce que l'on appelle les produits de la civilisation. Ils sont au service de certaines fonctions, exactement comme des organes, et le parallèle entre leur développement historique et le devenir phylogénétique des structures organiques prête fort à penser que, dans les deux cas, des facteurs analogues entrent en jeu, et surtout que c'est certainement la sélection et non pas la planification rationnelle qui joue là le rôle principal ${ }^{5}$.

5 De nombreuses critiques se sont alors élevées contre ces constructions historiques jugées entièrement artificielles, c'est-à-dire sans aucune conformité au réel. Le philosophe J.C.Beaune a ainsi dénoncé cette tentative d'assimiler le devenir technique à un processus naturel obéissant aux mêmes lois, mettant en œuvre les même mécanismes que l'évolution biologique ${ }^{6}$. Du côté de la sociologie, on oppose à ces reconstructions artificielles de s'interdire toute possibilité de commencer l'étude du processus effectif de l'innovation et du changement techniques. J.-C. Beaune approuve : le concept d'évolution technique est porteur d'une confusion entre la légalité sociale particulière qui à un moment donné sous-tend le changement, et "la légalité universelle d'un déterminisme inscrit dans la matière ». En se privant de tout contact avec la réalité des processus historiques, les théories de l'évolution technique procèderaient à des reconstructions sans aucun contenu de connaissance. La mise au 
travail de ce concept d'évolution technique ne s'accompagnerait d'aucune véritable production de connaissance sur les objets techniques.

6 Il est donc à première vue très embarrassant de découvrir une franche posture évolutionniste chez des auteurs qui ont incontestablement été animé par une intention de connaissance, et qui ont tout aussi incontestablement produit cette connaissance. Il en va ainsi du préhistorien Leroi-Gourhan, dont le legs en matière de connaissance technologique n'est pas discutable et qui néanmoins peut écrire :

dans leur très long développement... les techniques paraissent suivre le rythme de l'évolution biologique, et le chopper, le biface semblent faire corps avec le squelette. Au moment où émergent des possibilités cérébrales nouvelles, les techniques s'enlèvent dans un mouvement ascensionnel foudroyant, mais elles suivent des lignes qui miment à tel point l'évolution phylétique qu'on peut se demander dans quelle mesure elles ne sont pas l'exact prolongement du développement général des espèces ${ }^{7}$.

L'analyse des techniques montre que dans le temps elles se comportent à la manière des espèces vivantes... Il y aurait donc à faire une véritable biologie de la technique ${ }^{8}$.

7 La pensée de G. Simondon soulève la même difficulté. Simondon a une approche évolutionniste des techniques alors même qu'il est animé par l'intention de développer une connaissance technologique. T. Gaudin se réfère d'ailleurs à Du mode d'existence des objets techniques et à l'analyse de ce que Simondon appelle la concrétisation du moteur à combustion interne qui se dote d'ailettes de refroidissement. Du moteur de 1910, non pourvu d'ailettes, au moteur de 1956 qui en est au contraire pourvu, il y a un processus d'évolution que Simondon appelle concrétisation. Simondon explique par ailleurs que les objets techniques en voie de concrétisation se rapprochent du mode d'existence des êtres naturels (et il faut comprendre ici les êtres vivants) et que l'objet concret a des relations de causalité récurrente avec ce qu'il appelle un milieu associé, par analogie avec le milieu d'un être vivant. Il va même jusqu'à parler «d'évolution technique naturelle ». Situation inconfortable donc, au vu des critiques sans concession qui ont été faites de ce concept d'évolution technique et du rapprochement, jugé simplement métaphorique et sans valeur de connaissance, avec le domaine du vivant.

On peut alors montrer, et telle est la thèse soutenue ici, que la mise au travail de ce concept ne fait pas alternative avec un authentique projet de production de connaissance, bien au contraire. Non seulement le concept d'évolution technique n'est pas le fossoyeur d'une connaissance véritable des objets techniques et de leur devenir, mais il apparaît dans la pensée simondonienne (chez Leroi-Gourhan aussi) comme un concept-clé pour la construction de cette connaissance. En d'autres termes, le concept d'évolution ne rabat pas purement et simplement le domaine des objets techniques sur celui des êtres vivants, il ne vise pas à naturaliser les techniques en les coupant du domaine des significations humaines, il permet au contraire de constituer ce domaine des techniques en un domaine d'objectivité à part, autonome, irréductible et rendu accessible par une connaissance spéciale qui est la connaissance technologique. Simondon va même jusqu'à dire qu'en adoptant une vue évolutionniste des objets techniques, on se donne un accès à leur riche contenu de significations humaines. 


\section{L'analogie entre bioévolution et technoévolution. L'objet technique comme prolongement du corps}

9 Il convient tout d'abord de remarquer combien les évolutionnistes eux-mêmes, dès lors qu'ils sont animés par une authentique intention de connaissance, ont voulu mettre en garde contre toute idée de naturalisation des techniques. Ils appellent ainsi le plus souvent à manier avec beaucoup de prudence les analogies biologiques : selon LeroiGourhan, «dans le domaine de l'évolution technique, nous avons rencontré des faits qui peuvent s'organiser en images biologiques : ce n'est pas dire qu'ils sont du même ordre, mais simplement que la même réalité se retrouve ici et là dans les manifestations de la vie ${ }^{9}$; " "le progrès technique ne peut tout à fait être assimilé à une suite de mutations biologiques ${ }^{10} »$. De son côté, le philosophe évolutionniste F. Tinland explique qu'il ne faut pas aller jusqu'à parler d'une parenté effective entre technogenèse et organogenèse, que « la lame de silex n'engendre pas le couteau de cuivre et celui-ci n'engendre pas le sabre d'acier ${ }^{11} »$. On pourrait multiplier les citations de ce genre.

Prendre le contre-pied des anti-évolutionnistes n'est cependant pas une tâche facile tant ce concept a été galvaudé, détourné de son intention première qui est de rendre possible une connaissance rationnelle des outils. L'anthropologue anglais Pitt Rivers, qui a été selon B.Jacomy le premier à proposer au $\mathrm{xIX}^{\mathrm{e}}$ siècle de transposer l'évolutionnisme darwinien à l'étude des formes techniques, a ainsi entrepris de classer des outils selon un critère morphologique, en allant du plus simple au plus complexe. Le concept d'évolution technique a été forgé au départ comme un concept classificatoire, il a fonctionné comme une manière de classer les documents en vue d'une production de connaissance, et c'est comme tel qu'il a été mis au travail plus tard dans des études de technologie comme, par exemple, celles de C.Frémont ou d'A.LeroiGourhan. Certes ce concept a été récupéré par les discours d'accompagnement des techniques, parfois très peu préoccupés de connaissance scientifique (la publicité par exemple s'est aussi appropriée l'idée d'évolution technique ${ }^{12}$ ). Toutefois, son contenu de signification ne peut pas être réduit à ces récupérations.

11 La citation de T.Gaudin indique quels sont les trois points essentiels des approches évolutionnistes des techniques, et par conséquent quels sont les trois points sur lesquels porte la critique de ces approches: la première manière de comprendre l'analogie entre bioévolution et technoévolution consiste à assimiler les parties de l'objet technique à des organes, et l'objet lui-même à un organisme vivant ; la deuxième manière de comprendre cette analogie est de dire qu'ici et là les mécanismes du changement sont identiques (le devenir technique est régi par les mécanismes darwiniens de la variation et de la sélection naturelle ${ }^{13}$ ); la troisième manière de la comprendre consiste à affirmer que dans l'un et l'autre cas, il y a une autonomie de l'évolution par rapport à un sujet, par rapport à une intervention intelligente qui se représente des fins et qui planifie les transformations. Si cette affirmation est bien sûr au fondement d'une approche scientifique de l'évolution des êtres vivants, elle a été fortement contestée en ce qui concerne les techniques, notamment par tous ceux qui objectent qu'en laissant de côté la société des hommes, on bascule dans une mythologie de l'évolution (B.Latour) qui laisse non analysée et incomprise la dynamique véritable du changement technique.

Ces trois points doivent être discutés successivement. Toutefois, le premier contient dans une certaine mesure une ambiguïté qui peut déterminer la manière d'envisager 
les deux autres. En effet, il est possible de comprendre cette assimilation organologique de deux manières différentes: soit l'on dit que l'objet technique est analogue à un organisme fonctionnel et que des organes lui poussent ou s'atrophient ; soit l'on dit que l'objet technique est lui-même analogue à un organe, et l'on voit ici se profiler un thème célèbre affirmant que l'outil est le prolongement du corps, qu'il est chez l'homme l'analogue fonctionnel de ce que sont par exemple les griffes chez d'autres animaux. Les machines actuelles continuent alors le même processus et prolongent elles aussi le corps (selon l'idée par exemple que l'ordinateur prolonge le cerveau). Certains propos évolutionnistes ne semblent pas prêter à confusion sur ce point: comme dit Leroi-Gourhan, "l'Australanthrope paraît avoir possédé ses outils comme des griffes", "il semble les avoir acquis... comme si son cerveau et son corps les exsudaient progressivement ${ }^{14}$ ». Tout le processus de l'hominisation serait sous-tendu par une extériorisation progressive des fonctions biologiques, musculaires, sensorielles puis cérébrales, dans des objets techniques.

13 Ce thème de l'outil vu comme une émanation ou un prolongement du corps a été largement critiqué par des philosophes comme T. Adorno, M. Horkheimer ou encore $\mathrm{H}$. Arendt qui ont vu là une manière d'assimiler l'humanité à une espèce parmi les autres, ayant simplement mieux réussi que les autres. L'essentiel de la critique porte sur l'idée qu'en étant assimilé à un organe, à un prolongement du corps, l'objet technique doit nécessairement perdre sa réalité d'objet du monde.

L'espèce humaine, y compris ses machines, ses produits chimiques, ses énergies organisatrices - et pourquoi ne devrait-on pas considérer que tout cela fait partie d'elle comme les dents font partie de l'ours et rend les mêmes services en fonctionnant simplement encore mieux - représente à notre époque le dernier cri de l'adaptation ${ }^{15}$.

Considérés comme étant de même nature que les organes du corps, les objets techniques finissent par perdre leur statut d'objectivité pour devenir de simples prothèses ${ }^{16} \mathrm{du}$ corps, assimilables à des organes plus performants.

Telle est aussi la conclusion de $\mathrm{H}$. Arendt et de son analyse du processus de délabrement du monde des objets. L'existence d'un monde d'objets plus durables que chaque existence individuelle (le monde des œuvres) est selon Arendt une modalité de base de la condition humaine. Sans l'existence d'un monde d'objets durables, c'est-àdire non destinés à être consommés, l'humanité ne peut plus apparaître que comme une espèce qui a réussi. Que tout objet produit soit désormais destiné à être englouti dans le processus de la consommation, que toute activité soit aujourd'hui vouée à l'entretien du processus vital en lui fournissant les produits qu'il consomme, voilà le signe que l'homme est en train d'accepter :

de se changer en cette espèce animale dont, depuis Darwin, il s'imagine qu'il descend. À partir de ce moment, toutes les activités humaines n'apparaîtront plus que comme des processus et, par exemple, la motorisation moderne apparaîtrait comme un processus de mutation biologique dans lequel les corps humains commencent graduellement à se couvrir de carapaces d'acier ${ }^{17}$.

Les voitures dans lesquelles nous voyageons et que nous savons être construites par nous auront l'air, ainsi que Heisenberg l'a dit une fois, d'être une partie aussi inaliénable de nous-mêmes qu'une coquille d'escargot pour son occupant... La technologie entière... aura en fait cessé d'apparaître comme le résultat d'un effort conscient pour étendre sa puissance matérielle, mais plutôt comme un processus biologique à grande échelle ${ }^{18}$. 
16 L'homme a besoin du monde de l'artifice pour manifester son humanité. Considérer l'objet technique comme un prolongement du corps est donc le corollaire d'un délabrement de l'objectivité du monde et de l'assimilation toujours plus poussée de l'humanité à une espèce animale parmi d'autres. En assimilant l'objet technique à une réalité pour ainsi dire naturelle, l'évolutionnisme technique le ferait disparaitre comme objet détaché du corps, inassimilable au processus biologique (H. Arendt) et point d'appui pour une authentique manifestation de l'existence humaine.

\section{2. Évolution technique et construction de la connaissance technologique chez Leroi-Gourhan}

17 Or, il se trouve que l'approche de l'outil comme exsudation ou prolongement du corps n'a pas du tout pour but de le priver de sa consistance d'objet du monde, détaché du corps et autonome par rapport au processus biologique. Toute l'entreprise de LeroiGourhan consiste au contraire à faire progresser la seule connaissance qui puisse conférer à l'outil un statut d'objectivité véritable. Les outils sont une exsudation du corps, néanmoins «ils ne sont pas de même nature que mes ongles" précise LeroiGourhan. Dire que l'Australanthrope semble avoir possédé ses outils comme des griffes, cela ne veut pas dire que l'outil perd son statut d'objet du monde pour devenir un organe; cela veut dire que l'objet technique n'est pas à l'origine une production de l'intelligence, que l'Australanthrope ne l'a pas inventé dans un éclair génial et qu'au début la technique est un fait zoologique, une solution au grand problème de l'adaptation du vivant à son milieu. L'outil n'est pas un organe au sens de la griffe, c'est-à-dire qu'il est bel et bien un objet détachable du corps, extérieur à lui. C'est en tant que solution à un problème biologique que l'outil est assimilable à la griffe : cette assimilation ne lui enlève pas son objectivité, son caractère détachable. C'est même, comme l'a très bien dit B. Stiegler, dans l'amovibilité de l'outil que Leroi-Gourhan a cherché le moteur du processus de l'hominisation.

Toute la démarche de Leroi-Gourhan consiste à construire une connaissance technologique en donnant à cette connaissance son objet propre. À la question : quel est l'objet de la technologie ?, il semble qu'une réponse évidente puisse être donnée : c'est l'objet technique que nous avons sous les yeux, par exemple un galet taillé exhumé sur un site de fouilles. Selon Leroi-Gourhan, de cet objet isolé toutefois nous ne pouvons pas vraiment produire une connaissance. Nous ne pouvons en faire un objet de connaissance qu'en le resituant dans un processus d'évolution qui permet de mettre en évidence des régularités de structure, des lois de transformation dans ces structures. Ce processus n'a pas d'emblée une signification historique et Leroi-Gourhan insiste à plusieurs reprises sur la nécessité, en technologie, de distinguer le point de vue de l'histoire du point de vue de l'évolution. L'analyse de la planche des objets contondants est instructive sur ce point. Les différentes techniques de datation permettent par exemple d'affirmer que le chopping tool est chronologiquement antérieur au biface. Il est toutefois possible de classer les vestiges dans un ordre qui n'est pas celui de la chronologie mais celui de la logique fonctionnelle. Concernant la grande étude de technologie des années quarante, Évolution et techniques, Leroi-Gourhan explique ainsi que son intention n'est pas de faire une histoire des techniques, mais bien une mise en ordre logique des documents. Le critère retenu est celui de la tendance technique : le biface incarne mieux que le chopping tool la tendance, comme dit Leroi-Gourhan, à 
« couper n'importe quoi ». L'objet de la connaissance technologique n'est donc pas ici l'outil isolé, le silex proprement dit, mais le geste opératoire qui le manipule pour attaquer la matière. Il faut, dit Leroi-Gourhan, renoncer «à l'objet, en particulier à l'outil, [pour l'insérer dans] une formule force + matière = outil... qui donne l'objet extériorisé comme une sorte de dialogue, plus riche que la classification purement morphologique d'un outillage ${ }^{19}$ ». L'objet de la technologie, ce n'est pas l'outil mais le geste opératoire. On comprend mieux de ce point de vue en quel sens Leroi-Gouhran parle de tendance technique et de déterminisme technique : la tendance n'est pas une puissance souterraine qui pousse les techniques à se transformer, indépendamment des choix humains; elle est un concept classificatoire, logique, elle fournit un critère logique pour la mise en ordre des documents. Dans le cas des objets tranchants, ce critère est la coordination opératoire entre la structure du geste et celle de la matière. Plus le geste outillé parvient à coordonner ses opérations en adéquation avec les propriétés du matériau, et plus l'outil sera dit évolué. Leroi-Gourhan parle de déterminisme technique en ce sens : de même que Cuvier put prédire la mise au jour des os marsupiaux à partir de la structure de la mâchoire de sarigue, de même il est possible jusqu'à un certain point de «tirer de la forme d'une lame d'outil des prévisions sur celle du manche et sur l'emploi de l'outil complet». L'outil est la réponse à un problème précis qui n'admet qu'un nombre limité de solutions. "C'est parce que l'homme n'a pas d'autres prises sur le bois qu'en le coupant sous un certain angle, sous une pression déterminée, que les formes, les emmanchements des outils sont classifiables». Le déterminisme technique n'implique pas l'idée d'un autodéveloppement historique des techniques, indépendamment des choix humains; il signifie simplement que le geste opératoire a des contraintes structurales (liées notamment aux propriétés des matériaux), et que ces contraintes se traduisent par exemple par un nombre limité de possibilité d'associer une lame et un manche s'il s'agit d'un outil tranchant.

L'évolution technique n'est pas ici un concept historique, il ne s'agit pas du tout de fournir un modèle explicatif du changement technique dans l'histoire : il s'agit plutôt d'un concept logique, classificatoire, permettant la mise en ordre des documents de manière à rendre possible une connaissance, et ceci malgré notre ignorance des contextes sociaux de production et d'usage des premiers outils, malgré aussi le caractère très lacunaire des documents que nous possédons.

L'outil tranchant devient donc l'objet d'une connaissance technologique dès lors qu'on peut l'analyser du point de vue du geste opératoire et de ses contraintes, c'est-à-dire du point de vue de la tendance technique qui s'incarne successivement dans les différents objets tranchants. C'est par conséquent la possibilité d'insérer l'outil dans une série évolutive qui rend possible la connaissance technologique, c'est-à-dire la connaissance des stéréotypes techniques et des transformations dans la structure du geste opératoire. Le concept d'évolution technique ne dissout pas l'objectivité technique dans les processus naturels : il est au contraire un concept-clé dans la construction de cette objectivité. 


\section{L'évolutionnisme de Simondon. Le processus de concrétisation}

21 C'est exactement ce qu'explique Simondon dans le premier chapitre de la première partie de Du mode d'existence des objets techniques. Ce chapitre est en bonne partie consacré à la détermination du domaine d'objectivité de la connaissance technologique, et des concepts de cette connaissance. De quoi la connaissance technologique est-elle la connaissance? Quel est son objet propre ? Pour Simondon, comme pour Leroi-Gourhan, l'objet de la connaissance technologique, ce n'est pas tel objet isolé et donné dans l'expérience immédiate (par exemple, tel moteur à combustion interne); c'est la répartition des fonctions entre les différentes structures de l'objet, c'est-à-dire sa systématique fonctionnelle, et le processus qui a produit cette systématique par transformation du mode de coordination des différentes opérations techniques. L'objet de la technologie, c'est donc là aussi un processus d'évolution qui n'est pas un processus historique mais un processus régi par des lois de transformation de nature opératoire, fonctionnelle.

22 L'exemple de l'évolution du moteur à combustion interne est éclairant. Simondon revient deux fois sur cet exemple, une première fois pour évoquer l'apparition des ailettes de refroidissement sur la culasse des cylindres, une deuxième fois pour évoquer l'invention du moteur Diesel.

Les ailettes de refroidissement ont été ajoutées aux culasses des cylindres dans le but d'améliorer l'évacuation de la chaleur de l'intérieur vers l'extérieur des chambres de combustion, en augmentant la surface des échanges thermiques. Les ingénieurs se sont toutefois aperçus que l'adjonction d'ailettes avait aussi pour effet de renforcer la structure métallique du cylindre, soumis à la forte pression des gaz internes. Il a donc été possible de diminuer l'épaisseur des cylindres, ce qui a eu pour effet d'améliorer encore l'évacuation de la chaleur. Simondon parle dans ce cas d'un processus de convergence au sens où les ailettes remplissent leur fonction selon deux ordres d'effets physiques différents, un effet thermique d'abord (augmentation de la surface des échanges thermiques entre l'intérieur et l'extérieur des cylindres), un effet mécanique ensuite (consolidation de la structure métallique des cylindres et possibilité de réduire l'épaisseur métallique des cylindres). Ce processus de convergence qui rend les ailettes pluri-fonctionnelles et les différentes parties du moteur mieux corrélées entre elles, Simondon l'appelle concrétisation.

Le moteur Diesel concrétise autrement le moteur à combustion interne. Dans un moteur à essence à quatre temps, la carburation a lieu à l'extérieur du cylindre (dans le carburateur), le mélange air/gaz est ensuite admis dans le cylindre, il est porté à haute pression et donc à haute température par la remontée du piston, qui achève son cycle précédent, au point mort haut, le mélange est enflammé par un arc électrique (c'est le rôle des bougies), il explose et pousse le piston vers le bas. En remontant le piston évacue les gaz par la soupape d'échappement. Le piston revient au point mort bas, du mélange air/gaz est à nouveau admis, le piston entraîné par sa course remonte vers le point mort haut et un nouveau cycle a commencé. Dans le moteur Diesel, le carburant est directement admis dans le cylindre au moment où le piston est remonté au point mort haut (en achevant son cycle précédent). Le cylindre contient donc de l'air sous pression, c'est-à-dire porté à très haute température. L'injection directe de carburant dans l'air chaud provoque l'explosion et la détente du piston. Le moteur Diesel est en 
un sens plus simple que le moteur à essence, dans la mesure où il a été possible d'éliminer le carburateur et les bougies: l'injection directe de carburant est plurifonctionnelle, elle assure à la fois la carburation (qui a lieu dans le cylindre) et l'inflammation du mélange. Les opérations techniques sont mieux coordonnées, mieux corrélées entre elles. Par ailleurs, l'élévation de la température du mélange air/gaz quand il est comprimé par le piston n'a pas véritablement de rôle fonctionnel dans le moteur à essence. Il peut même être un effet nuisible au bon fonctionnement du moteur (si le mélange est trop chaud, il n'explose pas). C'est pourquoi il faut une limite au taux de compression. Dans le cas du moteur Diesel au contraire, cet effet lié à la compression intervient à titre de condition essentielle du fonctionnement. Simondon appelle concrétisation ce processus par lequel un effet du fonctionnement devient une condition essentielle de ce fonctionnement.

Historiquement, le moteur à combustion interne s'est donc concrétisé de deux façons différentes (au moins). La série évolutive n'a pas une signification directement historique. Le concept d'évolution par concrétisation est un concept technologique. Le critère d'évolution est le mode de coordination opératoire qui définit la systématique fonctionnelle de l'objet. Simondon explique alors qu'un objet technique en voie de concrétisation se rapproche du mode d'existence des êtres naturels et devient de plus en plus semblable à un organisme fonctionnel. Cela ne veut pas dire que l'objectivité technique se défait dans l'assimilation de l'être technique aux êtres biologiques. Cela veut dire au contraire que l'objet technique gagne en objectivité, c'est-à-dire en autonomie et en consistance. Le moteur dans lequel chaque structure est conçue pour accomplir une fonction déterminée est un agrégat, que Simondon appelle un objet technique abstrait. Un objet dans lequel au contraire les structures sont plurifonctionnelles (comme les ailettes qui ont une fonction thermique et une fonction mécanique), dans lequel se crée une meilleure coordination entre les opérations techniques, est un objet qui ressemble de plus en plus à un organisme par opposition au simple agrégat. Cette analogie est purement opératoire. C'est elle qui donne sa signification à l'idée que l'élément porteur de technicité peut être comparé à « ce qu'est un organe dans un corps vivant ${ }^{20} "$, comparaison qui se trouve immédiatement limitée à une signification fonctionnelle: Simondon précise en effet que l'élément technique, contrairement à l'organe biologique, est détachable et transposable d'un ensemble technique à un autre ensemble technique.

Cette idée, Simondon la prend dans une longue tradition de pensée. On la trouve notamment chez J. Lafitte, qui explique lui aussi qu'il y a une analogie entre l'élément technique et l'organe. L'intérêt de l'analogie toutefois n'est pas dans la pure et simple assimilation du technique au biologique. Il ne s'agit pas de naturaliser le domaine technique. Le corps vivant et la machine sont "formés, dit Lafitte, de cellules élémentaires, qui constituent des éléments organiquement indécomposables et jouissent de propriétés fonctionnelles singulières ». Dans l'histoire des techniques comme dans celle des formes vivantes, le processus évolutif est tantôt «de différenciation de fonctions s'isolant en organes spéciaux qui entraînent une complexité augmentée de l'organisation ", tantôt de "dégradation, de disparition, de déplacement de certains organes, entraînant des variations correspondantes dans les fonctions ». L'assimilation de l'organe biologique et de l'élément technique est de nature purement fonctionnelle ou opératoire, elle signifie qu'il est possible d'établir des 
analogies dans les manières dont, ici et là, dans le technique et dans le biologique, les structures se répartissent entre les fonctions qu'elles accomplissent.

Au-delà de l'analogie entre élément technique et organe, Simondon pousse la comparaison de l'objet technique qui se concrétise et d'un organisme vivant jusqu'à parler de l'existence d'un milieu associé de cet objet. Or, là encore il faut se garder de croire que la référence au milieu est simplement métaphorique. Elle est technologique, elle porte sur des considérations purement opératoires.

L'exemple de la turbine Guimbal illustre très bien ce point précis. Pour équiper les centrales hydroélectriques ${ }^{21}$, il s'est révélé intéressant de réduire en taille la turbine et la génératrice pour les immerger dans la conduite forcée, mais pour cela il fallait résoudre le problème de l'évacuation de la chaleur (une génératrice trop petite explose sous l'effet de la chaleur qu'elle dégage). La démarche d'invention de Guimbal a consisté à considérer le problème comme résolu dès le départ et à imaginer le fonctionnement effectif de la machine. La génératrice est plongée dans un carter d'huile et reliée par un axe à la turbine. Plus la machine tourne vite, plus elle chauffe, mais plus elle tourne vite, plus elle agite l'huile et plus celle-ci évacue rapidement la chaleur vers les parois du carter. L'eau a aussi une fonction de refroidissement, elle évacue la chaleur du carter. La turbine Guimbal est donc une machine qui intègre les propriétés des éléments naturels extérieurs (eau et huile) dans sa systématique fonctionnelle, dans ses schèmes opératoires. Elle renforce sa systématique fonctionnelle, comme dit Simondon elle l'axiomatise, dès lors que s'établit une coordination opératoire entre les éléments de la machine et les éléments du milieu. La concrétisation technique est par conséquent le processus par lequel se crée un ensemble fortement coordonné d'opérations, de telle façon que les effets induits par le fonctionnement de la machine sur le milieu extérieur (ici, l'agitation du bain d'huile) deviennent des éléments ayant une grande importance opératoire dans ce fonctionnement. A priori il n'y a rien de commun entre le fonctionnement de la génératrice et les propriétés thermiques de l'huile. Le processus de concrétisation consiste à rendre commensurable ce qui ne l'était pas au départ, c'est-à-dire ici à établir une coordination opératoire entre les éléments de la machine et les éléments du milieu extérieur.

La concrétisation des objets techniques rétrécit par conséquent l'intervalle entre sciences et techniques. L'objet concret est l'objet qui est connu de manière à peu près identique selon l'intention constructive et selon le regard scientifique. Dans le cas de la turbine Guimbal, une démarche abstraite d'invention aurait consisté par exemple à essayer de réduire en taille la génératrice, indépendamment des effets physiques de son fonctionnement sur le milieu. La machine est connue selon l'intention constructive (fabriquer une machine réduite en taille et qui n'explose pas), mais elle n'est pas connue selon l'ensemble des phénomènes physico-chimiques dont elle va être le siège une fois qu'elle fonctionnera. Essayer au contraire de faire coïncider l'intention constructive et la connaissance de ces phénomènes, de telle sorte qu'idéalement chaque phénomène soit interprété d'un point de vue opératoire, et devienne partie intégrante des schèmes de fonctionnement de la machine : voilà ce qu'est le processus de concrétisation par établissement d'une relation de causalité récurrente (opératoire) entre la machine et son milieu associéé2. 


\section{Conclusion} leur soi-disant entreprise de naturalisation, de biologisation de la technique, entendue comme une perte d'objectivité. Toute l'analyse de Simondon rejoint celle de LeroiGourhan sur au moins un point très précis : le processus d'évolution technique est le processus par lequel la relation directe entre l'homme et la nature devient de moins en moins anthropocentrique et de plus en plus opératoire; le processus d'évolution technique est le processus par lequel la relation à la nature s'objective en se formalisant sous la forme d'un ensemble de mieux en mieux coordonné d'opérations. Il y a bien une relation à la nature impliquée dans la turbine Guimbal, néanmoins cette relation est intérieure à l'objet technique lui-même, elle est dans la coordination opératoire qui définit son organisation, dans la systématique fonctionnelle qui régit son fonctionnement. En d'autres termes, cette relation à la nature acquiert un caractère d'objectivité, décentré par rapport au thème de l'homme qui est face à la nature et la transforme au moyen des médiations techniques ${ }^{23}$. Comme dirait Piaget, le processus d'évolution technique fait passer de l'égocentrique à l'opératoire, en décentrant les opérations techniques par rapport au point de vue de l'action directe de l'homme sur la matière. Le concept d'évolution est ainsi un concept-clé dans la construction de l'objectivité technique, il permet d'aborder objectivement l'être technique en le détachant du thème anthropologique, c'est-à-dire du point de vue de l'homme qui s'outille pour intervenir dans la nature.

\section{BIBLIOGRAPHIE}

Adorno Theodor, Horkheimer Max, La dialectique de la raison. Fragments philosophiques, Paris, Gallimard, 1974.

Arendt Hannah, Condition de l'homme moderne, Paris, Calmann-Lévy, 1961.

Arendt Hannah, La crise de la culture, Paris, Gallimard, 1972.

Basalla George, The Evolution of Technology, Cambridge University Press, 1988.

Beaune Jean-Claude, La technologie introuvable, Paris, Vrin, 1980.

Dreyfus Hubert, L'intelligence artificielle. Mythes et limites, Paris, Calmann-Lévy, 1979.

Gaudin Thierry, De l'innovation, La Tour d'Aigues, Éditions de l'Aube, 1998.

Latour Bruno, Aramis ou l'amour des techniques, Paris, La Découverte, 1992. 
Leroi-Gourhan André, Évolution et techniques. Milieu et techniques, Paris, Albin Michel, 1973.

Leroi-Gourhan André, Les racines du monde. Entretiens avec Claude-Henri Rocquet, Paris, P. Belfond, 1982.

Leroi-Gourhan André, Le geste et la parole. Technique et langage, Paris, Albin Michel, 1989.

Minsky Marvin, “Machines are More than They Seem”, Science Journal, vol. 4, nº 10, oct. 1968, p. 3-43.

Simondon Gilbert, Du mode d'existence des objets techniques, Paris, Aubier, 1989.

Tinland Franck, La différence anthropologique. Essai sur les rapports de la nature et de l'artifice, Paris, Aubier-Montaigne, 1977.

\section{NOTES}

1. Marvin Minsky, "Machines are More than They Seem ", Science Journal, vol. 4, n 10, oct. 1968, p. 3 ; propos cité par Hubert Dreyfus, L'intelligence artificielle. Mythes et limites, Paris, CalmannLévy, 1979.

2. Voir Bruno Latour, Aramis ou l'amour des techniques, Paris, La Découverte, 1992.

3. Ibid., p. 11

4. Thierry Gaudin, De l'innovation, La Tour d'Aigues, Éditions de l'Aube, 1998.

5. Ibid., p. 58-62.

6. Jean-Claude Beaune, La technologie introuvable, Paris, Vrin, 1980.

7. André Leroi-Gourhan, Le geste et la parole. Technique et langage, Paris, Albin Michel, 1989, p. 152.

8. Ibid., p 206-207.

9. André Leroi-Gourhan, Évolution et techniques. Milieu et techniques, Paris, Albin Michel, 1973, p. 436-437.

10. André Leroi-Gourhan, Les racines du monde. Entretiens avec Claude-Henri Rocquet, Paris, P. Belfond, 1982, p. 171.

11. Franck Tinland, La différence anthropologique. Essai sur les rapports de la nature et de l'artifice, Paris, Aubier-Montaigne, 1977, p. 160.

12. Un spot publicitaire présentait voici quelques années le couteau suisse comme le point d'aboutissement d'une évolution commencée avec les premiers outils de silex, en passant par de nombreuses formes transitoires. On voyait le silex se transformer en une série d'outils pour finalement devenir couteau suisse.

13. Voir par exemple George Basalla, The Evolution of Technology, Cambridge University Press, 1988.

14. André Leroi-Gourhan, Le geste et la parole. Technique et langage, p. 151.

15. Theodor Adorno, Max Horkheimer, La dialectique de la raison. Fragments philosophiques, Paris, Gallimard, 1974, p. 238-239.

16. En un sens évidemment différent de celui que Bernard Stiegler donne à ce mot: la prothéticité chez lui ne fait pas perdre aux êtres techniques leur statut d'objets extérieurs au corps, au contraire.

17. Hannah Arendt, Condition de l'homme moderne, Paris, Calmann-Lévy, 1961, p. 401.

18. Hannah Arendt, La crise de la culture, Paris, Gallimard, 1972, p. 354-355.

19. André Leroi-Gourhan, Évolution et techniques. L'homme et la matière, p. 318-319.

20. Gilbert Simondon, Du mode d'existence des objets techniques, Paris, Aubier, 1989, p. 65.

21. Les groupes bulbes de l'usine marémotrice de la Rance sont du type Guimbal. 
22. Simondon précise au demeurant qu'une machine n'est jamais concrète, il y a toujours un résidu d'abstraction au sens où elle ne peut pas être connue absolument identiquement selon l'intention fabricatrice et selon le regard scientifique.

23. Ce thème de la médiation technique ne disparaît pas complètement chez Simondon, mais il est profondément remanié.

\section{RÉSUMÉS}

Une abondante littérature critique a interprété le concept d'évolution technique dans le sens d'une «naturalisation» des techniques. Une approche évolutionniste de la technique impliquerait une conception purement biologique des techniques. De ce point de vue les techniques ne seraient plus décrites comme des faits sociaux et comme des objets du monde humain, mais comme des prolongements des fonctions biologiques apparaissant et disparaissant au gré d'un processus d'évolution «naturelle». Or, à rebours de ces critiques, on peut montrer que parler d'évolution technique n'équivaut pas ipso facto à dissoudre le technique dans le biologique. Une théorie évolutionniste de la technique peut au contraire être motivée par l'intention de délimiter la technique comme un domaine à part entière du savoir et de la culture, irréductible au domaine vital. On le montre en s'appuyant sur les travaux de Leroi-Gourhan et Simondon.

Does a theory of "technological evolution" prompt the idea that technology is a natural phenomenon? The analogy between natural evolution and technological change has been often outlined in paleoanthropology and in various general theories of machines since the $19^{\text {th }}$ century. Does this analogy mean that technology is embedded in a purely natural and even biological process? Beyond its social and cultural features, technology would be viewed as an output of a biological evolution process, as the "externalization" of biological functions. Against this approach to "technological evolution" as a purely biological vision of technology, we argue that this concept has been shaped in order to delineate an autonomous field of human activity and knowledge, irreducible to biology. The article focuses on French paleontologist A.LeroiGourhan's and French philosopher G.Simondon's theories of “technological evolution”.

\section{INDEX}

Personnes citées : Simondon (Gilbert), Leroi-Gourhan (André)

Mots-clés : évolutionnisme, objectivité, technique

\section{AUTEUR}

\section{XAVIER GUCHET}

Maître de conférences, Paris I 